\title{
Videoscopic adrenalectomy - when does retroperitoneal seem better?
}

Piotr Myśliwiec ${ }^{1}$, Magda Marek-Safiejko², Jerzy Łukaszewicz ${ }^{1}$, Kamil Safiejko ${ }^{1}$, Robert Kozlowski², Piotr Wojskowicz ${ }^{1}$, Jacek Dadan ${ }^{1}$

${ }^{1} 1^{\text {st }}$ Department of General and Endocrinological Surgery, Medical University of Bialystok, Poland

${ }^{2}$ Department of Oncological and General Urology, Bialystok Sniadecki Memorial Provincial Hospital, Bialystok, Poland

Videosurgery Miniinv 2014; 9 (2): 226-233

DOI: $10.5114 /$ wiitm.2014.41636

\begin{abstract}
Introduction: Videoscopic adrenalectomy is the gold standard for the surgical treatment of benign adrenal tumours. The two most common approaches are the lateral transperitoneal approach (LTA) and the posterior retroperitoneal approach (PRA). So far it has not been established which is the preferable one. The choice depends primarily on the surgeon's preference and contraindications for a particular approach in individual cases.

Aim: To compare the two most common approaches for videoscopic adrenalectomy, LTA and PRA, based on a literature review and our own experience.

Material and methods: We reviewed the literature (PubMed and Cochrane 1990 - current) and analysed retrospectively our own patients who underwent videoscopic adrenalectomy between 2009 and 2013. The analysis covered patient features, tumour characteristics, reasons for qualification for each approach, operative time and postoperative complications.

Results: Out of 71 videoscopic adrenalectomies, 50 were performed using PRA and 21 using LTA. Patients in the PRA group on average were older, but had smaller tumours. There was no significant difference in perioperative morbidity between PRA and LTA. Mean blood loss and total operative time were smaller/shorter for the PRA group in comparison to the LTA group. Time to first oral intake and the average postoperative hospital stay were shorter for PRA than LTA.

Conclusions: Videoscopic adrenalectomy is a safe and efficient technique performed using both LTA and PRA approaches. The choice of technique should be guided, most of all, by surgeon experience, patient characteristics, tumour size and location. Our preferable approach is PRA, especially for small $(<6 \mathrm{~cm})$ benign tumours. We find LTA advantageous for tumours of larger size, with suspected malignant character (either primary or secondary), in ectopic position, as well as in patients lacking an ipsilateral kidney or when a simultaneous abdominal operation is planned.
\end{abstract}

Key words: videoscopic adrenalectomy, retroperitoneal, adrenal tumours, laparoscopic surgery.

\section{Introduction}

Lately, we have been witnessing intense development of laparoscopic surgery, which is less invasive than classical interventions, and involves fewer complications. Paired adrenal glands are small, frail and located deep inside the adipose tissue of the retroperitoneal area; hence, their adequate exposure is challenging. Open adrenalectomy requires a large incision, quite disproportionate to the size of the organ involved [1]. Therefore, since Gagner described the first laparoscopic adrenalectomy in 1992, the

\section{Address for correspondence:}

Kamil Safiejko MD, $1^{\text {st }}$ Department of General and Endocrinological Surgery, Medical University of Bialystok, 24a M. Skłodowskiej-Curie St, 15-276 Bialystok, Poland, phone: +48 508859 993, e-mail: kamil.safiejko@gmail.com 
technique has rapidly gained popularity and is now recognised as the gold standard for the treatment of tumours smaller than $6 \mathrm{~cm}[2,3]$.

Clinical trials conducted in various centres worldwide have confirmed the technological capacity, safety and efficacy of the laparoscopic method [4]. In comparison with the open approach, laparoscopy requires longer operative time at the early phase of training, but is associated with less postoperative pain, shorter hospital stay, faster recovery, reduced mortality, less intraoperative bleeding, a better cosmetic effect and lower costs [4, 5].

As a result of continuous development of laparoscopy, obesity, age, circulatory and respiratory diseases are no longer contraindications to such interventions. Operative injury reduction made it easier for the elderly, obese or those suffering from circulatory diseases to qualify for treatment.

There are four methods of accessing adrenal glands: anterior transperitoneal, lateral transperitoneal, lateral retroperitoneal, and posterior retroperitoneal approaches. The two most popular approaches are the lateral transperitoneal approach (LTA) and posterior retroperitoneal approach (PRA), described by Mercan in 1995 and popularised by Martin Walz [6].

A new trend in videoscopic surgery is to minimise the number of access sites and to reduce the size of the operative wound. This is where single-port interventions originated [7]. The Da Vinci Surgical System was also employed in videoscopic adrenalectomy in all approaches. It significantly increased the cost of the procedures with no benefits over conventional PRA or LTA.

\section{Indications and contraindications for laparoscopy}

In the case of small $(\leq 6 \mathrm{~cm})$, benign adrenal tumours, videoscopic treatment is the gold standard $[1,4]$. The technique is most often used to remove adenoma known to cause Conn's syndrome, Cushing's syndrome, pheochromocytoma (PCC) or hormonally inactive incidentaloma.

Pheochromocytoma, a rare tumour of chromaffin cells secreting catecholamines, may result in haemodynamic disturbances due to manipulation in the adrenal gland area during an operative procedure [8]. Said complications have disqualified patients with PCC from videoscopic treatment for a long time. Nowadays, it is known that videoscopic adrenalecto- my is feasible, safe [9-12] and may successfully be employed in over $90 \%$ of pheochromocytomas [13]. The technique is associated with a decreased complication rate, and fewer negative haemodynamic consequences and perioperative hypertensive incidents when compared to classical procedures [11, 12]. A prerequisite for a safe intervention is preoperative pharmacotherapy inhibiting tumour hormonal activity and an experienced surgical-anaesthetic team.

Thanks to innovative technologies and growing surgical experience, large tumours $(>6 \mathrm{~cm}$ ) and malignant lesions, previously restricted to classical interventions only, are increasingly reported to be removed videoscopically. Laparoscopic excision of tumours larger than $8 \mathrm{~cm}$ is associated with a prolonged operative time, increased blood loss and longer hospitalisation period, as compared to smaller tumours. However, perioperative morbidity remains comparable to adrenalectomies for smaller masses [14]. Tumours exceeding $10 \mathrm{~cm}$ in diameter should probably be operated on in a classical manner [1, 11].

If the results of diagnostic imaging suggest a malignant lesion infiltrating into surrounding tissues, classical adrenalectomy is indicated. However, in the case of malignant tumours of 4-6 cm, which seem not to infiltrate into surrounding tissues, as evidenced by diagnostic imaging, selected patients may benefit from minimally invasive adrenalectomy [15].

In the case of a rare, but highly aggressive, adrenocortical carcinoma (ACC), adrenalectomy is the only potentially effective treatment [16]. Recently, there have been some reports on a successful stage I and II ACC resection with the application of the videoscopic technique [17]. Porpiglia et al. [18] compared open and videoscopic adrenalectomy in the case of ACC, finding no difference between the two as per the postoperative recurrence rate, which at the same time invalidated the theory of increased risk of disease recurrence and dissemination of cancer cells following videoscopic treatment. However, decisions to operate on those patients videoscopically need to be taken cautiously, considering the devastating outcome of ACC.

Adrenal glands are areas of metastasis development for mammary, pulmonary, skin and renal tumours in particular. Often, the sole purpose of operative treatment in the course of the above is to extend the life of the patient [19], as it usually does not cure the neoplastic disease. If a negative margin can be achieved during adrenalectomy, the video- 
scopic technique, dubious initially, has now become a standard treatment procedure for such lesions as it is associated with lower mortality and a low recurrence rate, comparable to open adrenalectomy [20].

The resection of both ACC and adrenal metastases should be performed by highly qualified surgeons, as it may be necessary to convert to the classical method if difficulties or complications occur during the procedure.

\section{Aim}

The study aimed to compare the two most common approaches for videoscopic adrenalectomy, LTA and PRA, based on a literature review and our own experience.

\section{Material and methods}

We reviewed the literature (PubMed and Cochrane 1990 - current) for the following key words: videoscopic adrenalectomy, laparoscopic adrenalectomy, retroperitoneal adrenalectomy retroperitoneoscopy.

The clinical analysis included patients treated in the $1^{\text {st }}$ Department of General and Endocrinological Surgery, Medical University of Bialystok (UMB), between February 2009 and April 2013, who underwent videoscopic adrenalectomy. Most patients were prepared for operations in the Department of Endocrinology, where the functional status of adrenal glands was evaluated, and where diagnostic imaging (CT) was performed. Every patient suspected of having adrenal carcinoma or tumour $>12 \mathrm{~cm}$ was qualified for the classical approach surgery, and as such was excluded from this analysis. 43 females and 28 males aged 34 to 79 (mean age: 56.7) were operated on.

The analysis covered patient characteristic features, tumour morphological characteristics, reasons for qualification for the PRA and LTA surgery, operative time, perioperative blood loss and postoperative complications. Non-parametric tests were used for statistical analysis.

\section{Recommended videoscopic access methods}

For LTA, the patient is positioned in the lateral decubitus position on the side that is opposing the adrenal gland involved (Photo 1 ). The operating table is flexed at the waist to open up the space between the iliac crest and the ribs. Because of relocation of the skin against the costal arch when placing the patient in a lateral position, it is well worth marking the border of the costal arch before doing so. The first $10 \mathrm{~mm}$ trocar is introduced $3-4 \mathrm{~cm}$ below the ribs - at the extension of the midclavicular line. Pneumoperitoneum is insufflated to $12-15 \mathrm{~mm} \mathrm{Hg}$. Then, two $5 \mathrm{~mm}$ trocars are placed $6-8 \mathrm{~cm}$ laterally to the optical trocar.

During left adrenalectomy it is necessary to incise the spleno-renal and spleno-diaphragmatic ligaments. Thanks to gravity, the free falling spleen and pancreatic tail expose the adrenal gland. Sometimes, the left adrenal gland is difficult to locate due to abundant retroperitoneal fat and a small tumour. A laparoscopic ultrasound device was reported as useful in such cases. Next, the left adrenal gland is exposed and dissected.

On the right side, care must be taken to avoid injury to the liver, especially when the organ is pushed

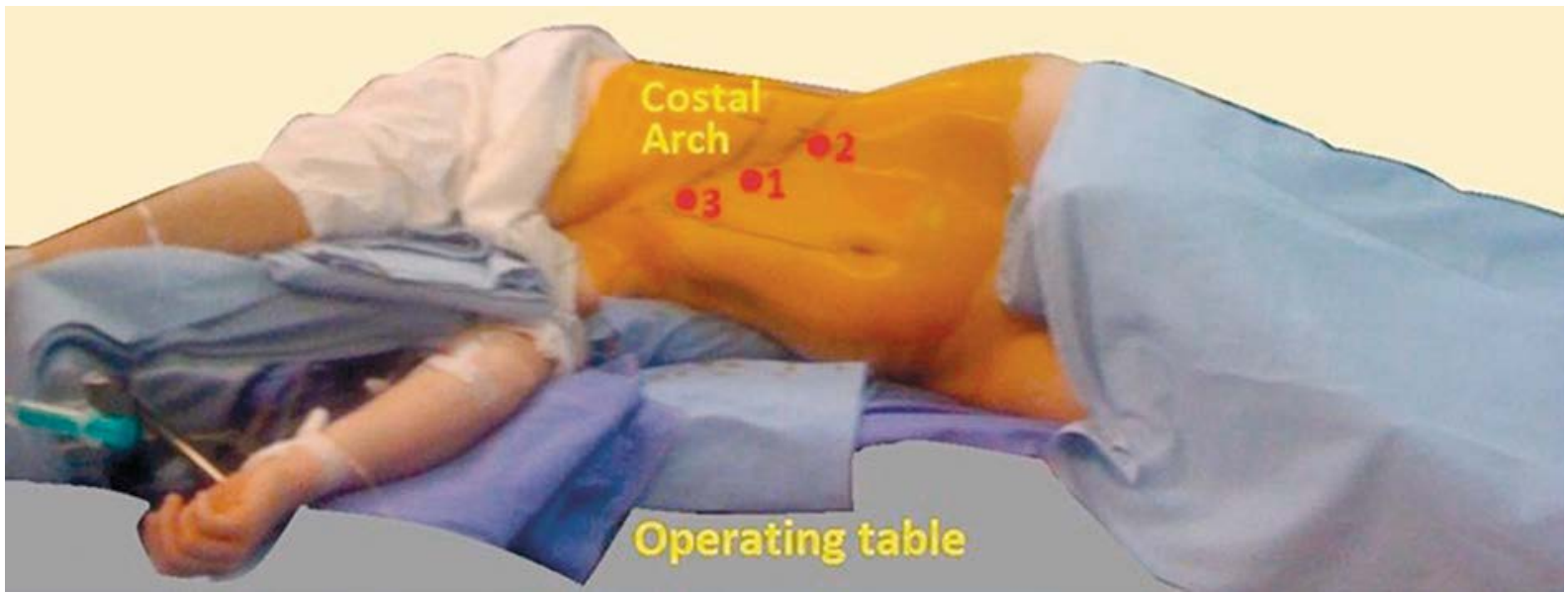

Photo 1. Position of the patient during lateral transperitoneal adrenalectomy (author Piotr Myśliwiec) 


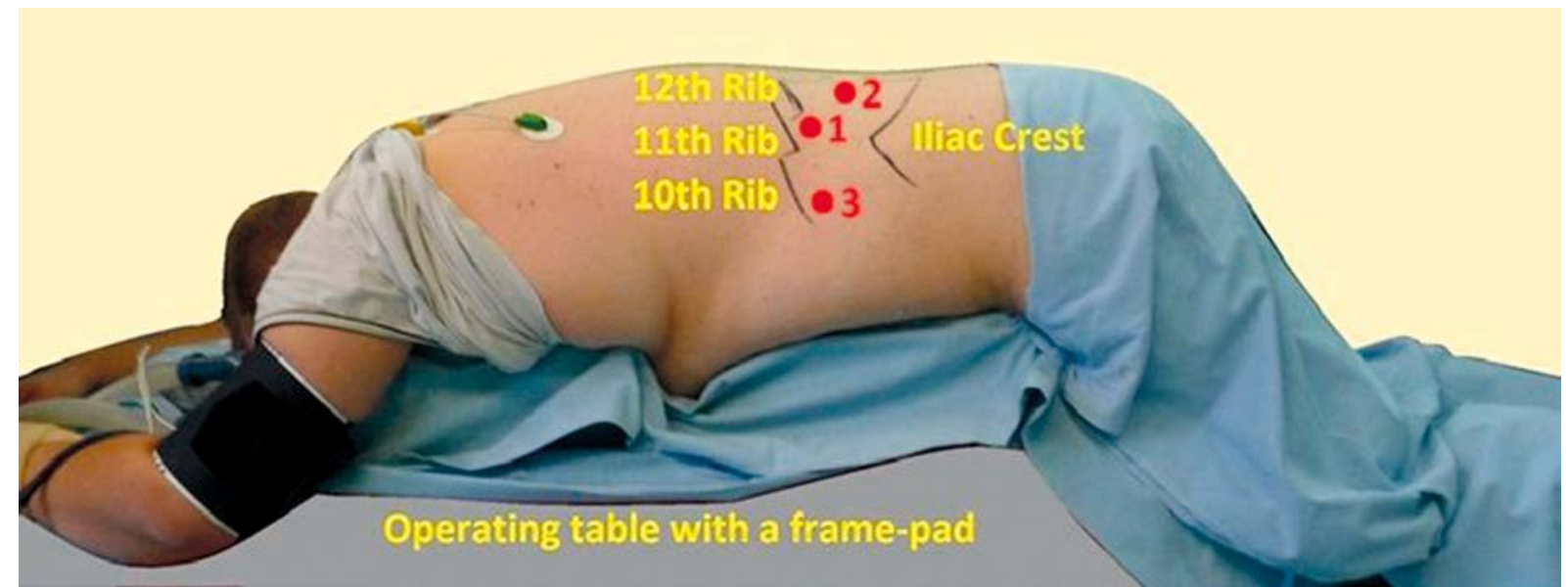

Photo 2. Position of the patient during posterior retroperitoneal adrenalectomy (author Piotr Myśliwiec)

forward by an enlarged adrenal gland. In our experience the Hasson technique is advisable. An additional trocar is necessary to introduce the liver retractor. The procedure starts with freeing the right hepatic triangular ligament and retracting the liver. Upon identification of the right kidney, Gerota's fascia is incised. When the inferior vena cava, which runs in the direct vicinity of the adrenal gland, is dissected, the central adrenal vein requires precise identification and securing. Next, additional right adrenal veins and arteries are sealed and the gland is dissected and removed in an extraction bag.

Posterior retroperitoneal approach usually begins with intubation on the trolley. The patient is then transferred onto the operating table and placed on his abdomen (Photo 2). The trunk of the patient is fitted on a rectangular foam frame, which makes the belly hang loose. Lower limbs are flexed at hip and knee joints. In such a position the space between the $12^{\text {th }}$ rib and the posterior superior iliac spine expands, which facilitates access to the retroperitoneal space. For unilateral PRA, the operated side can be slightly elevated. A 2-cm long dissection at the tip of the $12^{\text {th }}$ rib allows blunt creation of retroperitoneal access. Guided by the index finger, a $10-\mathrm{mm}$ trocar is introduced paravertebrally ca. $3-4 \mathrm{~cm}$ beneath the $12^{\text {th }}$ rib. The trocar and the skin should form a $45^{\circ}$ angle in order to avoid difficulties when operating the camera, especially in obese or muscular patients. Then, a $5-\mathrm{mm}$ trocar is introduced underneath the $11^{\text {th }}$ rib, $\sim 6 \mathrm{~cm}$ laterally. A trocar fitted with a balloon to prevent gas escape is placed in the initial wound. $\mathrm{CO}_{2}$ is insufflated to $20-24 \mathrm{~mm} \mathrm{Hg}$, or even $28 \mathrm{~mm} \mathrm{Hg}$ in obese patients [21]. Dissection of the tumour-affected adrenal gland begins with finding the upper renal pole and then it is continued medially, between the crura of the diaphragm and the adrenal gland. Main adrenal vessels are located in this position. The vein and artery are dissected and sealed with clips or a vessel-sealing device. Subsequently, the adrenal gland with the tumour is dissected free and removed in the extraction bag. The use of percutaneous ultrasound pre- or perioperatively, particularly in the case of massive retroperitoneal adipose tissue, has been described [22].

\section{Lateral transperitoneal approach or posterior retroperitoneal approach?}

To date, there has been no prospective, randomised study comparing both approaches. Thus, neither was proven superior. The choice depends primarily on the surgeon's preference and contraindications for a particular approach in an individual case.

The majority of surgeons perform videoscopic adrenalectomy using LTA. It is the result of better expertise in the anatomy of the peritoneal cavity, greater skills acquired during other laparoscopic interventions, and a large operative field. Lateral transperitoneal approach allows for easier removal of some atypically located, larger adrenal gland tumours [23]. Additionally, this approach enables a potential simultaneous operation on other organs located intraperitoneally [24, 25].

Currently, PRA is becoming more and more common. Posterior retroperitoneal approach gives the 
most direct access to the adrenal gland without the need to mobilise intraperitoneal organs. Bilateral tumours can be operated on using PRA at the same time, without changing position [22]. In patients with a history of abdominal surgery, this approach provides an unaffected space free from adhesions. Some data suggested shorter operative time, less blood loss, shorter hospital stay and a comparable number of complications in the case of PRA, when compared to LTA; however, there is not enough evidence to prove the superiority of one technique over the other [21, 26-30]. A breakthrough for PRA was an increase in $\mathrm{CO}_{2}$ pressure from the standard 12-15 mm Hg up to 20-24 mm Hg, or even $28 \mathrm{~mm} \mathrm{Hg}$ [21], which enlarged the operative field. What is more, there is better haemostasis of the operative area due to vessel compression. Opponents of the method have suggested that increased $\mathrm{CO}_{2}$ pressure creates a risk of gas embolism and ejection of catecholamines [31]. Those findings have not been confirmed in practice $[14,22]$.

Even though videoscopic techniques employing PRA and LTA pertain to the same organ, they are completely different surgical procedures that require separate training. According to some, the learning curve in the case of PRA is shorter and requires only 20 procedures [22].

A less common videoscopic technique is the lateral retroperitoneal approach. A prospective, randomised clinical trial including 57 patients and comparing this approach to LTA found no significant differences in the operative time, estimated blood loss or complication rate [32]. Also, the amount of analgesics, time needed for patient mobilisation, return to oral feeding and length of hospital stay were comparable in both techniques. Still, convalescence time was shorter in the case of the retroperitoneal approach [32].

\section{Results}

In the study period, 71 videoscopic adrenalectomies were performed, including 50 PRA and 21 LTA. A subtotal excision of the adrenal gland, where a morphologically unaffected part of the gland was left in situ, was performed 4 times - in patients after contralateral adrenalectomy and without suspicion of malignancy. The patient average ASA scale score was 2.56 (between 2 and 4). Body mass index (BMI) of patients who underwent surgery was with- in the range $22-44 \mathrm{~kg} / \mathrm{m}^{2}$ (mean $\mathrm{BMI}-29.9 \mathrm{~kg} /$ $\mathrm{m}^{2}$ ). Most PRA procedures (93\%) were performed by a single operator, with application of either the PRA or LTA.

All the adrenalectomies reported were primary operations. The predominant preoperative diagnosis was a hormonally inactive tumour. In 39 (55\%) cases, the tumour was located on the left, and in the remaining $32(45 \%)$ it was on the right. Two patients had a two-stage, bilateral adrenalectomy performed; one was operated twice using PRA, and the other had LTA on the right and PRA on the left side. For simplicity and because they underwent operations in different years, they are presented as " 4 separate" patients. Preoperative patient characteristics and the type of tumour are presented in Table I. The time of PRA operation ranged from $60 \mathrm{~min}$ to $170 \mathrm{~min}$ (mean time - $93 \mathrm{~min}$ ), whereas for LTA it was from $95 \mathrm{~min}$ to $240 \mathrm{~min}$ (169 min on average). The size of tumours excised using PRA in the specimen ranged from $1 \mathrm{~cm}$ to $7 \mathrm{~cm}$ (4.7 cm on average), and for LTA between $2 \mathrm{~cm}$ and $11 \mathrm{~cm}(7.6 \mathrm{~cm}$ on average). Hospitalisation lasted 1 to 5 days (mean 2.0 days) after PRA, and 2 to 5 days (mean 2.8 days) after LTA. The outcomes are displayed in Table II. The most common postoperative diagnosis was that of adenoma. Three cases involved adrenocortical carcinoma: one in the PRA group ( $3 \mathrm{~cm}$ in diameter) and two in the LTA group $(5.5 \mathrm{~cm}$ and $9 \mathrm{~cm}$ ). Metastasis to the adrenal gland was also observed three times in the series: one in the PRA group $(4.5 \mathrm{~cm}$ - of pulmonary origin) and two in the LTA group $(8 \mathrm{~cm}$ and $7 \mathrm{~cm}$, both of renal origin).

There were no mortalities. One patient operated on using LTA for phaeochromocytoma developed pulmonary oedema intraoperatively. The patient was hospitalised in the Intensive Care Unit, to be transferred to the Clinic on day 2, and discharged home on day 3. Inability of the patient to tolerate the full dose of $\alpha$ - and $\beta$-blockers in the preoperative period (the patient only tolerated $1 / 4$ of the prescribed dosage) could predispose to this complication. In 1 case, a negative re-videoscopy was performed on day 2 after PRA owing to fever of unclear origin - the patient (a heavy smoker) turned out to have acute bronchitis. One patient from the PRA group aged 72 developed pneumonia postoperatively. Three (6\%) PRA patients and 2 (10\%) LTA patients reported pain radiating to the hypogastric area, lasting more than 7 days. 
Table I. Patients' characteristics

\begin{tabular}{|c|c|c|c|}
\hline Parameter & PRA $(n=50)$ & $\operatorname{LTA}(n=21)$ & Value of $p$ \\
\hline Age, mean \pm SD & $55.6 \pm 9.3$ & $48.4 \pm 10.4$ & $<0.05$ \\
\hline Gender, $n(\%)$ : & & & NS \\
\hline Male & $19(38)$ & $9(43)$ & \\
\hline Female & $31(62)$ & $12(57)$ & \\
\hline BMI, mean $\pm \mathrm{SD}\left[\mathrm{kg} / \mathrm{m}^{2}\right]$ & $30.1 \pm 4.2$ & $27.9 \pm 3.8$ & NS \\
\hline ASA, median (min-max) & $2(2-4)$ & $3(2-4)$ & NS \\
\hline Tumour size, mean \pm SD & $4.7 \pm 1.3$ & $7.6 \pm 3.4$ & $<0.001$ \\
\hline Location, $n$ (\%): & & & NS \\
\hline Right side & $23(46)$ & $9(43)$ & \\
\hline Left side & $27(54)$ & $12(57)$ & \\
\hline Hormonal diagnosis, $n$ (\%): & & & $<0.05$ \\
\hline Pheochromocytoma & $12(24)$ & $4(19)$ & \\
\hline Cushing's disease & $5(10)$ & $3(14)$ & \\
\hline Conn's disease & $7(14)$ & $1(5)$ & \\
\hline Non-functioning tumour & $26(52)$ & $13(62)$ & \\
\hline Pathological diagnosis, $n(\%)$ : & & & $<0.01$ \\
\hline Adenoma & $33(66)$ & $12(57)$ & \\
\hline Pheochromocytoma & $12(24)$ & $4(19)$ & \\
\hline Other - benign & $3^{*}(6)$ & $1^{\#}(5)$ & \\
\hline Adrenocortical carcinoma & $1(2)$ & $2(9.5)$ & \\
\hline Metastasis & $1(2)$ & $2(9.5)$ & \\
\hline
\end{tabular}

PRA - posterior retroperitoneal approach, LTA - lateral transperitoneal approach, NS - not significant. *In the PRA group there were single cases of haematoma, hyalinization and fibrotic tissue. "In the LTA group there was one schwannoma

\section{Discussion}

Patients were qualified either for PRA or LTA based on diagnostic imaging results (ultrasound and computed tomography). In the case of tumours $<7 \mathrm{~cm}$, interventions were performed using PRA. For tumours $>7 \mathrm{~cm}$ or in patients without a kidney on the same side due to previous nephrectomy or agenesis, LTA was employed. The reason for avoiding large PRA for large tumours is a relatively small operative space, characteristic for this approach.

The first 31 consecutive videoscopic adrenalectomies were performed using PRA. With acquired experience, the time of adrenalectomies performed in our department using PRA decreased from 180 to $\sim 70 \mathrm{~min}$. Walz et al. scrutinised data regarding over
500 videoscopic adrenalectomies conducted with the application of PRA [21]. According to them, since 1992 the average operative time has shortened to 40 min, which makes the procedure comparable to cholecystectomy or laparoscopic hernia repair in terms of the time it takes. The above results have been confirmed by other studies [26].

To avoid intraoperative arterial pressure increase during surgery for phaeochromocytomas, we avoided tumour mobilisation before sealing of adrenal vessels. Intraoperative increase in arterial pressure $>170 \mathrm{~mm} \mathrm{Hg}$ was noted in 2 patients (16.7\%) operated on using PRA and in 1 patient (25\%) during LTA. Dickson et al. [28] reported similar results, recording an intraoperative arterial pressure rise in $17 \%$ of patients operated on using PRA. 
Table II. Operative outcomes $(n=71)$

\begin{tabular}{|c|c|c|c|}
\hline Parameter & PRA $(n=50)$ & $\operatorname{LTA}(n=21)$ & Value of $p$ \\
\hline Mean operative time, mean \pm SD [min] & $93 \pm 28.5$ & $168.7 \pm 43.8$ & $<0.001$ \\
\hline Blood loss, mean $\pm S D[\mathrm{ml}]$ & $58.6 \pm 62.3$ & $158.3 \pm 78.4$ & $<0.001$ \\
\hline First oral intake, mean \pm SD [days] & $1.0 \pm 0.2$ & $1.6 \pm 0.9$ & $<0.05$ \\
\hline Postoperative hospital stay, mean \pm SD [days] & $2.0 \pm 0.5$ & $2.9 \pm 0.9$ & $<0.01$ \\
\hline Conversions & $1^{a}$ & $1^{b}$ & \\
\hline Complications (acc. to Dindo) altogether, $n(\%)$ : & $8(16)$ & $6(29)$ & NS \\
\hline । & $4^{c}(8)$ & $2^{d}(10)$ & \\
\hline$\|$ & $3^{e}(6)$ & $3^{f}(14)$ & \\
\hline III & $1^{g}(2)$ & 0 & \\
\hline IV & 0 & $1^{\mathrm{h}}(5)$ & \\
\hline V & 0 & 0 & \\
\hline
\end{tabular}

PRA - posterior retroperitoneal adrenalectomy, LTA - lateral transperitoneal adrenalectomy. ${ }^{a}$ Conversion from PRA to LTA due to anaesthetist's request compression of intubation tube; ${ }^{b}$ conversion from LTA to open adrenalectomy after unsuccessful attempt of creating pneumoperitoneum acc. to Hasson's technique - due to bleeding from the liver extensively adherent to the anterior abdominal wall; ' 3 patients with pain radiating to hypogastric area lasting $>7$ days, 1 patient requiring physiotherapy for atelectasis; ${ }^{d} 2$ patients with pain radiating to hypogastric area lasting $>7$ days; ${ }^{e} 2$ patients obtained blood transfusions ( 1 or 2 units of red packed cells each), one developed pneumonia in postoperative period; $f 3$ patients obtained blood transfusions ( 2 units of red packed cells each); ${ }^{g} 1$ negative re-videoscopy was performed on day 2 after PRA due to fever of unclear origin; ${ }^{h} 1$ patient with pheochromocytoma treated for 2 days in ICU due to pulmonary oedema

The $\mathrm{CO}_{2}$ insufflation pressure created in the retroperitoneal space during PRA interventions was 20-28 $\mathrm{mm} \mathrm{Hg}$, as opposed to 12-15 mm Hg for LTA surgery. Higher insufflation led to higher end-expiratory $\mathrm{CO}_{2}$ tension. This was not accompanied by a rise in intraoperative blood pressure or breathing disturbances after surgery.

Even though no randomised clinical trial has demonstrated superiority of either videoscopic approach, it has been suggested that PRA entails less pain in the postoperative period. Also, the learning curve was reported to be shorter for PRA than LTA $[21,26]$. Our median PRA surgery time, $93 \pm 28.5 \mathrm{~min}$, and the continuing downward trend, confirm the above observation.

It remains dubious whether videoscopic methods should be used for malignant tumours which do not infiltrate the surrounding tissues in preoperative imaging. We made efforts to avoid tumours suspected of malignant proliferation when qualifying patients for videoscopic adrenalectomy. Based on histopathological analyses of removed tumours, six primary or secondary malignant adrenal tumours were identified. Follow-up examinations of 6-18 months after surgery did not show any evidence of recurrence.
Our initial experience demonstrates that videoscopic adrenalectomy is a safe and efficient technique performed using both LTA and PRA. Despite the fact that the mean age in the PRA group was higher and that BMI, ASA or tumour location differences were insignificant, tumour size and the rate of patients with hormonally active tumours were significantly higher in the LTA group. Therefore, the groups were not comparable. Nevertheless, pronounced differences were found in the operative time, amount of blood lost intraoperatively, time of return to oral feeding and the time of hospital stay between PRA and LTA surgery, to the advantage of PRA.

The choice of technique should be guided, most of all, by surgeon experience, patient characteristics and tumour size and location. Our preferable approach is PRA and we recommend it for small $(<6 \mathrm{~cm})$ benign adrenal tumours in particular. Posterior retroperitoneal approach makes it possible to remove both adrenal tumours simultaneously. For larger tumours, in patients lacking a kidney on the operated side, with tumours located high (difficult to reach from a retroperitoneal approach), ectopically, suspected to be malignant (peritoneum covering the adrenal gland may need removal) or when another laparoscopic procedure is needed, we opt for LTA. 


\section{References}

1. Ramacciato G, Mercantini P, La Torre M, et al. Is laparoscopic adrenalectomy safe and effective for adrenal masses larger than $7 \mathrm{~cm}$ ? Surg Endosc 2008; 22: 516-21.

2. Gagner M, Lacroix A, Bolte E. Laparoscopic adrenalectomy in Cushing's syndrome and pheochromocytoma. New Engl I Med 1992; 327: 1033.

3. Soon PS, Yeh MW, Delbridge LW, et al. Laparoscopic surgery is safe for large adrenal lesions. Eur I Surg Oncol 2008; 34: 67-70

4. Gill IS. The case for laparoscopic adrenalectomy. J Urol 2001; 166: 429-36.

5. Lee J, El-Tamer M, Schifftner T, et al. Open and laparoscopic adrenalectomy: analysis of the National Surgical Quality Improvement Program. J Am Coll Surg 2008; 206: 953-9.

6. Mercan S, Seven R, Ozarmagan S, et al. Endoscopic retroperitoneal adrenalectomy. Surgery 1995; 118: 1071-6.

7. Beiša V, Kryžauskas M, Simutis G, et al. Surgical treatment options for aldosteronomas. Videosurgery Miniinv 2012; 7: 260-7.

8. Fernandez-Cruz L, Taura P, Saenz A, et al. Laparoscopic approach to pheochromocytoma: hemodynamic changes and catecholamine secretion. World J Surg 1996; 20: 762-8.

9. Tiyadath BN, Sukumar S, Saheed CS, et al. Laparoscopic adrenalectomy. Is it any different in phaeochromocytoma and non-phaeochromocytoma? Asian J Surg 2007; 30: 244-9.

10. Zhang X, Lang B, Ouyang JZ, et al. Retroperitoneoscopic adrenalectomy without previous control of adrenal vein is feasible and safe for pheochromocytoma. Urology 2007; 69: 849-53.

11. Toniato A, Boschin IM, Opocher G, et al. Is the laparoscopic adrenalectomy for pheochromocytoma the best treatment? Surgery 2007; 141: 723-7.

12. Tiberio GA, Baiocchi GL, Arru L, et al. Prospective randomized comparison of laparoscopic versus open adrenalectomy for sporadic pheochromocytoma. Surg Endosc 2008; 22: 1435-9.

13. Shen WT, Grogan R, Vriens M, et al. One hundred two patients with pheochromocytoma treated at a single institution since the introduction of laparoscopic adrenalectomy. Arch Surg 2010; 145: 893-7.

14. Castillo OA, Vitagliano G, Secin FP, et al. Laparoscopic adrenalectomy for adrenal masses: does size matter? Urology 2008; 71: 1138-41.

15. Icard P, Goudet P, Charpenay C, et al. Adrenocortical carcinomas: surgical trends and results of 253 patient series from the French association of Endocrine Surgeons study group. World J Surg 2001; 25: 891-7.

16. Allolio B, Hahner S, Weismann D, et al. Management of adrenocortical carcinoma. Clin Endocrinol (Oxf) 2004; 60: 273-87.

17. McCauley LR, Guyen MM. Laparoscopic adrenalectomy for cancer: long term outcomes. Curr Opin Urol 2008; 18: 134-8.

18. Porpiglia F, Fiori C, Daffara F, et al. Retrospective evaluation of the outcome of open versus laparoscopic adrenalectomy for stage I and II adrenocortical cancer. Eur Urol 2010; 57: 873-8.

19. Kim SH, Brennan MF, Russo P, et al. The role of surgery in the treatment of clinically isolated adrenal metastasis. Cancer 1998; 82: 389-94.

20. Henry JF, Defechereux T, Raffaelli M, et al. Complications of laparoscopic adrenalectomy: results of 169 consecutive procedures. World J Surg 2000; 24: 1342-6.
21. Walz MK, Alesina PF, Wenger FA, et al. Posterior retroperitoneoscopic adrenalectomy - results of 560 procedures in $520 \mathrm{pa}$ tients. Surgery 2006; 30: 899-908.

22. Berber E, Tellioglu G, Harvey A, et al. Comparison of laparoscopic transabdominal lateral versus posteriori retroperitoneal adrenalectomy. Surgery 2009; 146: 621-5.

23. Assalia A, Gagner M. Laparoscopic adrenalectomy. Br J Surg 2004; 91: 1259-74.

24. Fiszer P, Toutounchi S, Pogorzelski R, et al. Is tumour size a contraindication to laparoscopic adrenalectomy? Videosurgery Miniinv 2012; 7: 144-6.

25. Gagner M, Pomp A, Heniford BT, et al. Laparoscopic adrenalectomy: lessons learned from 100 consecutive procedures. Ann Surg 1997; 226: 238-46.

26. Lee CR, Walz MK, Park S, et al. A comparative study of the transperitoneal and posteriori retroperitoneal approaches for laparoscopic adrenalectomy for adrenal tumors. Ann Surg Oncol 2012; 19: 2629-34.

27. Li QY, Li F. Laparoscopic adrenalectomy in pheochromocytoma: retroperitoneal approach versus transperitoneal approach. J Endourol 2010; 24: 1441-5.

28. Dickson PV, Alex GC, Grubbs EG, et al. Posterior retroperitoneoscopic adrenalectomy is a safe and effective alternative to transabdominal laparoscopic adrenalectomy for pheochromocytoma. Surgery 2011; 150: 452-8.

29. Beiša V, Kildušis E, Strupas K, et al. Single access retroperitoneoscopic adrenalectomy: initial experience. Videosurgery Miniinv 2012; 7: 45-9.

30. Myśliwiec P, Dadan J, Łukaszewicz J. Two tumours of the right adrenal gland treated videoscopically by the posterior retroperitoneal approach. Videosurgery Miniinv 2009; 4: 126-30.

31. Flavio Rocha M, Faramarzi-Roques R, Tauzin-Fin P, et al. Laparoscopic surgery for pheochromocytoma. Eur Urol 2004; 45: 226-32.

32. Rubinstein M, Gill IS, Aron M, et al. Prospective, randomized comparison of transperitoneal versus retroperitoneal laparoscopic adrenalectomy. J Urol 2005; 174: 442-5.

Received: 3.07.2013, accepted: 31.08.2013. 